\title{
La flexibilidad curricular para la participación de las Familias en Educación Infantil
}

\section{Curricular flexibility for the participation of Families in Early Childhood Education}

\author{
Manuel Delgado-García ${ }^{1}$, José Antonio Ruíz Rodríguez ${ }^{2}$ \\ ${ }^{1}$ Universidad de Huelva manuel.delgado@dedu.uhu.es \\ ${ }^{2}$ Universidad de Huelva joseantonio.ruiz@dedu.uhu.es
}

Recibido: $29 / 8 / 2020$

Aceptado: $10 / 12 / 2020$

\section{Copyright (C)}

Facultad de CC. de la Educación y Deporte. Universidad de Vigo

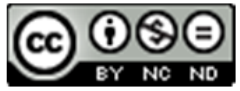

Dirección de contacto:

Manuel Delgado García

Facultad de Educación, Psicología y Ciencias del Deporte

Campus de "El Carmen"

Avda. de las Fuerzas Armadas, s/n

21007 Huelva

\begin{abstract}
Resumen
La participación de la familia en la etapa de Educación Infantil es un factor de especial relevancia para el proceso educativo en su conjunto. Para atender los fundamentos de esta premisa, desde este trabajo se establecen dos objetivos fundamentales: analizar la opinión de las familias con hijos/as en la etapa de Educación Infantil para identificar en qué se focalizan las acciones flexibles que promueven los centros educativos y determinar la influencia de las variables "tiempo de relación con el centro" y "nivel educativo de los estudiantes" en relación a las consideraciones sobre la flexibilidad curricular de los centros educativos. A partir de los planteamientos del paradigma cuantitativo, se desarrolla una investigación de tipo encuesta, apoyada en un diseño de tipo descriptivo y correlacional, no experimental y de corte transversal, en la que 88 familias son encuestadas. Como conclusiones, las familias reconocen la existencia de medidas o acciones que flexibilizan el currículo de Educación Infantil y que les permiten ser partícipes del proceso educativo (en mayor medida se intensifica la relación en familias que llevan menos tiempo de relación con el centro), aunque se limita en gran medida a aspectos de menor impacto pedagógico.
\end{abstract}

\section{Palabras clave}

Relación Familia-Escuela, Educación Infantil, Investigación Curricular, Participación Familiar, Organización Escolar

\begin{abstract}
Family participation in the Early Childhood Education stage is a factor of special relevance for the educational process as a whole. To address the foundations of this premise, from this work two fundamental objectives are established: to analyze the opinion of families with children in the early childhood education stage to identify what the flexible actions promoted by educational centers are focused on and to determine the influence of the variables "time of relationship with the center" and "educational level of the students" in relation to the considerations on the curricular flexibility of the educational centers. Based on the approaches of the quantitative paradigm, a survey-type research is developed, supported by a descriptive and
\end{abstract}


correlational, non-experimental and cross-sectional design, in which 88 families are surveyed. As conclusions, families acknowledge the existence of measures or actions that make the infant education curriculum more flexible and that allow them to participate in the educational process (the relationship is more intensified in families that have been in contact with the center for less time), although it is largely limited to aspects of less pedagogical impact.

\section{Key Words}

Family School Relationship, Early Childhood Education, Curriculum Research, Family Involvement, School Organization

\section{INTRODUCCIÓN}

La implicación de las familias en la educación tiene un único objetivo común: la educación de sus hijos. Y es en la etapa de Educación Infantil donde esa implicación sobresale sobre otras etapas, por ello es adecuado realizar desde un primer momento una flexibilidad curricular, donde la participación activa y el reconocimiento de la familia a lo que se hace en el centro sea proclive al desarrollo de cada una de las áreas del niño mediante la realización de una serie de actividades (Zabalza y Formosinho, 1998).

Esta participación, enmarcándola desde un punto de vista legislativo, se postula en el artículo 27, apartados 5 y 7 de la Constitución Española como derecho constitucional, y se recoge en la LOMCE (2013), que ha visto reducida la participación de las familias en relación a Leyes educativas anteriores, dando más protagonismo a los directores de centro, estableciéndose por ello, desde la Organización para la Cooperación y el Desarrollo Económico (OCDE), la Unión Europea o el Consejo de Europa la implicación de las familias como un requisito imprescindible para la calidad de los sistemas educativos (Egido-Gálvez, 2015)

Una participación de las familias derivada desde las escuelas a través de diferentes medios informativos, tanto unidireccionales (circulares, paneles de anuncios, web, blogs, etc.) como bidireccionales (entrevistas individuales, agenda escolar, reuniones grupales, etc.) (Macia-Bordalba, 2017), fomentando por ello los logros en el niño o la niña en su rendimiento académico, siendo el sentimiento de pertenencia al centro y las expectativas académicas los ejes de dicho logro (Ministerio de Educación, Cultura y Deporte-MECD en adelante-, 2014); existen estudios que reseñan que las familias que están mejor informadas sobre el comportamiento de sus hijos en la escuela alcanzan mejor rendimiento (MECD, 2015).

Es el centro el que debe proponer, por tanto, actuaciones o programas para que las familias expresen sus preocupaciones y experiencias, fomentando estrategias educativas (Colegio Concertado Nuestra Señora de la Providencia -CNSP en adelante-, 2015), estableciendo contactos asiduos sobre el desarrollo del menor (Ruiz-De Miguel y García-García, 2004), acordes en su proceso de enseñanza y aprendizaje desde los 3 o cuatro años (González-Valenzuela, Martín-Ruiz, Prieto-Adánez y Rivas-Moya, 2018).

¿Y cómo debe ser esa relación familia-centro? Esa relación ha de estar ubicada en el mesosistema (Bronfenbrenner, 1979, 2005), lugar donde se mide la frecuencia en las relaciones y el nivel de implicación de las familias (Arranz-Freijo, Olabarrieta-Artetxe, Manzano-Fernández, Martín-Ayala, Galende-Pérez, 2012), representando el ámbito más 
influyente en el desarrollo del menor (Burns, 1990; De Vicente-Yagüe y GonzálezRomero, 2019; Rivas y Ugarte, 2014; Ruiz-de Miguel, 1999), compartiendo responsabilidad educativa (Ruiz-de Miguel, 1999), generando intercambios de información de esas relaciones (Sierra-Martínez, 2018), mejorando el logro académico en el niño (Serrano-Muñoz, Mérida-Serrano y Tabernero-Urbieta, 2016) y siendo a día de hoy un reto educativo (Garreta, 2008; Macia-Bordalba, 2017).

En relación a esto, es necesario establecer una serie de conexiones entre los centros educativos y las familias, así como con los tiempos escolares (Vendrell-Mañós, GeisBalagué, Anglès-Virgili y Dalmau-Montalà, 2019), como pueden ser las relaciones con el profesorado, la inclusión de eventos, el voluntariado o los grupos de discusión (MECD, 2014). Pero es indudable que toda forma de participación no debe priorizarse en aquellas actividades que se realizan dentro de la educación formal (Payá-Rico, 2010; Vendrell-Mañós et al., 2019), sino en todas aquellas actividades preescolares que fomenten la enseñanza y el aprendizaje (Ortega-Ruiz, Romera y Monks, 2013).

Muchas son las actividades y actuaciones que se llevan a cabo en los centros en la etapa de Educación Infantil y que flexibilizan el currículo, donde el juego es considerado una parte primordial para que el niño pueda desarrollarse individual y colectivamente (Jover y Payá-Rico, 2013). Un juego que puede realizarse mediante la educación formal o la no formal. Estas actividades pueden girar en torno al desarrollo de un área determinada o a una serie de competencias básicas atendiendo a la flexibilización curricular. Si atendemos a la competencia TIC, considerada como una de las experiencias que mejor se vinculan con el niño hoy en día (Rideout, Vandewater y Wartella, 2003; Robles-Meléndez, 2012), algunas de estas actividades se relacionan con el booktrailer o narrativas digitales (Ibarra-Rius y Ballester-Roca, 2016), el uso del videojuego digital y educativo (Sampedro-Requena, Muñoz-González y Vega-Gea, 2017), la enseñanza de la programación en informática (Da Silva-Filgueira y GonzálezGonzález, 2017; González-González, 2019), el trabajo con imágenes familiares (Esteban-Guitart, Oller y Vila, 2012) o mediante dramatizaciones (Aristizábal, Lasarte y Tressserras, 2015), así como con el empleo de la Fotovoz (Doval, Martínez-Figueira y Raposo, 2013).

En cuanto a la capacidad de comunicación lingüística, el cuentacuentos es una de las actividades que más se utilizan en esta etapa como práctica educativa (Amar, 2018), como en los casos del Kamishibai (Cid-Lucas, 2009), la casa y la familia (El Ouarit, Bouhachi y Eddaif, 2007), el Programa Success for All en EEUU (Santos-Rego y Slavin, 2002) o el aprendizaje a través de la escritura inventada (Alves-Martins, Salvador, Albuquerque y Montanero-Fernández, 2017). También se desarrolla la competencia matemática a través de un programa de intervención virtual para la mejora de la misma (Alzubi, Fernández, Flores, Durán y Cotos, 2018; Durán, Álvarez, Fernández y Acuña, 2015; Fernández-Abella, Peralbo-Uzquiano, Durán-Bouza, BrenllaBlanco y García-Fernández, 2019) o el estímulo de la creatividad (Franco-Justo y JustoMartínez, 2010).

$\mathrm{Si}$ atendemos a actividades menos formales, algunas de las actividades son las visitas a los museos con la familia (Laboratorio Permanente de Público de Museos, 2016), la participación en actividades extraescolares (Arranz- Freijo et al., 2012), actividades de carácter lúdico o actuaciones como reuniones generales con la familia, entrevistas individuales o actividades de ocio, entre otros (Tamayo, 2014), la inclusión de un gabinete psicopedagógico (CCNSP, 2015), tertulias literarias, programas de desarrollo 
del lenguaje oral, ludoteca, incremento del tiempo escolar, etc. (CEIP Antonio Machado -CEIP AM en adelante-, 2015), protocolos de comunicación con las familias, tutorías activas, visita del profesorado a las casas, voluntariado, biblioteca, formación de adultos, asambleas, programas de refuerzo, entre otras (Consejo Escolar de Estado CEE en adelante-, 2015).

En ese sentido, muchas son las experiencias que se llevan a cabo en diferentes centros educativos con multitud de actividades y que se resumen en la siguiente tabla (Tabla 1).

\begin{tabular}{ll}
\hline \multicolumn{1}{c}{ CENTRO } & \multicolumn{1}{c}{ ACTIVIDADES } \\
\hline $\begin{array}{l}\text { Colegio Privado Concertado Cardenal Espínola, } \\
\text { Linares, Jaén. }\end{array}$ & $\begin{array}{l}\text { Tertulias dialógicas, actividades de aula, tutorías } \\
\text { activas, cooperación de la Policía Local, la } \\
\text { resolución de conflictos, entre otras }\end{array}$ \\
\hline $\begin{array}{l}\text { Colegio Público Ramón Sainz de Varanda, } \\
\text { Zaragoza. }\end{array}$ & $\begin{array}{l}\text { Programa Ajedrez en la Escuela, Biblioteca escolar, } \\
\text { cuentacuentos, proyecto leer juntos, mercadillo } \\
\text { solidario, etc. }\end{array}$ \\
\hline Colegio Público la Pradera, Valsaín, Segovia. & $\begin{array}{l}\text { Galardonado con el Premio Nacional de Educación } \\
\text { en 2013 por el funcionamiento del centro y la } \\
\text { participación de las familias }\end{array}$ \\
\hline Colegio Público Entre Culturas, Hellín, Albacete. & $\begin{array}{l}\text { Café-tertulia con charlas sobre hábitos y rutinas en } \\
\text { Educación Infantil }\end{array}$ \\
\hline CEIP Balaídos, Vigo. & $\begin{array}{l}\text { Desarrollo de la CreAPPtividad en Educación } \\
\text { Infantil }\end{array}$ \\
\hline CEIP Valdespartera II, Zaragoza. & $\begin{array}{l}\text { Actividades sobre 3D y la realidad aumentada en } \\
\text { Educación Infantil }\end{array}$ \\
\hline CEIP Isidoro Andrés Villarroya, Castellón. & $\begin{array}{l}\text { Proyecto colaborativo "Els continents", sobre los } \\
\text { animales, la realidad aumentada y el Croma Key }\end{array}$ \\
\hline CEIP Nuestra Señora del Carmen, Murcia & $\begin{array}{l}\text { Proyecto la vuelta al mundo, con especial hincapié } \\
\text { en los dioses egipcios }\end{array}$ \\
\hline
\end{tabular}

Tabla 1. Actividades ofertadas en los centros educativos. Fuentes: MECD (2015); MedinaFuentes (2019); Molinero-Maldivo (2019); Pardo-Artero (2019); Santabárbara-Bayo (2019).

Es indudable que esta flexibilización curricular va a depender de cómo se lleve a cabo esa relación entre la familia y la escuela, es decir, del nivel de implicación de las familias, del centro, del alumnado, de las vías de comunicación entre ellas, de la transparencia que exista, de las actividades que se realicen, tanto las formales como otras menos formales y la clave de todo esto reside en la etapa de Educación Infantil, que es el momento en el cual la implicación de la familia tiene más incidencia en el rendimiento del alumnado.

\subsection{Objetivos}

Esta investigación tiene como objetivo principal identificar qué variables relacionadas con la flexibilidad curricular potencian los centros educativos de Educación Infantil para favorecer la implicación de las familias en la educación de sus hijos y mejorar la respuesta educativa que ofrecen.

Para su consecución se establecen otros objetivos específicos:

1. Analizar la opinión de las familias con hijos/as en la etapa de Educación Infantil para identificar en qué se focalizan las acciones flexibles que promueven los centros educativos. 
2. Determinar la influencia de las variables "tiempo de relación con el centro" y "nivel educativo de los estudiantes" en relación a las consideraciones sobre la flexibilidad curricular de los centros educativos.

Las hipótesis que guiarán el desarrollo del estudio y que atienden a los objetivos son las siguientes:

- Hipótesis $1\left(\mathrm{H}_{1}\right)$ : las familias afirman que los centros educativos ofrecen múltiples acciones que flexibilizan el currículo oficial para favorecer su participación (CCNSP, 2015; CEIP AM, 2015; Jurado-Gómez, 2009; MECD, 2014, 2015; Tamayo; 2014).

- Hipótesis $2\left(\mathrm{H}_{2}\right)$ : las familias reconocen que la flexibilidad curricular se relaciona en mayor medida con aspectos ligados al plano de la organización del centro y las orientaciones próximas al proceso de enseñanza y aprendizaje del estudiante (Castro-Zubizarreta y Manzanares-Ceballos, 2016; CCNSP, 2015; Ceballos-López y Saiz-Linares, 2019; MECD, 2014).

- Hipótesis $3\left(\mathrm{H}_{3}\right)$ : las familias que poseen una mayor relación temporal con los centros educativos son aquellas que reconocen la existencia de más medidas de flexibilidad curricular (García-Hernández, 2003; Hernández-Prados, GomarizVicente, Parra-Martínez y García-Sanz, 2015, 2016; MECD, 2014).

- Hipótesis $4\left(\mathrm{H}_{4}\right)$ : las familias cuyos hijos se encuentran matriculados en el primer ciclo de Educación Infantil son destinatarias de un mayor número de acciones flexibles para lograr mejorar su participación en los centros educativos (Garreta, 2008; Gomariz, Parra, García-Sanz, Hernández y Pérez, 2008; MECD, 2014, 2015; Paniagua-Valle, 2009; Parra, Gomariz, Hernández-Prados y García-Sanz, 2017; Peñalver-Pérez, 2009).

\section{MÉTODO}

A partir de los planteamientos del paradigma cuantitativo se desarrolla una investigación de tipo encuesta, apoyada en un diseño de tipo descriptivo y correlacional, no experimental y de corte transversal.

\subsection{Participantes}

La población tomada como referencia para el objeto de estudio hace referencia a las familias con hijos/as matriculados en la etapa de Educación Infantil en centros educativos públicos de la provincia de Huelva (España). La selección de los participantes en el estudio se realiza a partir de un muestreo no probabilístico en el que se utiliza como criterio de selección la accesibilidad y disposición a participar de manera voluntaria en el estudio, así se accede a una muestra de 88 familiares $(72,7 \%$ mujeres; $27,3 \%$ hombres), de los que el 27,3\% tiene a hijos matriculados en el primer ciclo de Educación Infantil (Centros de Educación Infantil de 0 a 3 años de edad) y el $72,7 \%$ los tiene matriculados en el segundo ciclo de Educación Infantil (Centros de Educación Infantil y Primaria de 3 a 6 años de edad); el 60,2\% tiene una edad entre los 31 y 41 años $(\bar{x}=2,83)$ y son unidades familiares en su mayoría conformadas por parejas con hijos/as (46,6\%) o Monoparentales (madre o padre) con hijos/as (42\%) y en las que el tiempo de relación con el centro se muestra muy disperso: Menos de 1 año $(17,0 \%)$; 
Entre 1 y 2 años (37,5\%); Entre 3 y 5 años (26,1\%); Más de 5 años $(19,3 \%)(\bar{x}=2,48$; $\mathrm{sd}=.99)$. Otras características sociodemográficas están recogidas en la Tabla 2.

\begin{tabular}{|c|c|c|}
\hline Variable & Valores & Porcentajes \\
\hline \multirow{4}{*}{$\begin{array}{c}\text { Situación laboral } \\
\text { del encuestado }\end{array}$} & Trabajo a jornada completa & $35,6 \%$ \\
\hline & Trabajo a tiempo parcial & $35,6 \%$ \\
\hline & Sin trabajo & $27,6 \%$ \\
\hline & Otro & $1,1 \%$ \\
\hline \multirow{4}{*}{$\begin{array}{c}\text { Situación laboral } \\
\text { del cónyuge }\end{array}$} & Trabajo a jornada completa & $60 \%$ \\
\hline & Trabajo a tiempo parcial & $25,9 \%$ \\
\hline & Sin trabajo & $12,9 \%$ \\
\hline & Otro & $1,2 \%$ \\
\hline \multirow{6}{*}{$\begin{array}{l}\text { Nivel de estudios } \\
\text { del encuestado/a }\end{array}$} & Menos de estudios primarios & $1,1 \%$ \\
\hline & Estudios primarios completos, certificado escolar & $17 \%$ \\
\hline & Educación Secundaria Obligatoria & $17 \%$ \\
\hline & Bachillerato & $8 \%$ \\
\hline & Formación Profesional (Grado Medio o Superior) & $21,6 \%$ \\
\hline & Estudios Universitarios & $35,2 \%$ \\
\hline \multirow{6}{*}{$\begin{array}{l}\text { Nivel de estudios } \\
\text { del cónyuge }\end{array}$} & Menos de estudios primarios & $1,2 \%$ \\
\hline & Estudios primarios completos, certificado escolar & $27,9 \%$ \\
\hline & Educación Secundaria Obligatoria & $15,1 \%$ \\
\hline & Bachillerato & $11,6 \%$ \\
\hline & Formación Profesional (Grado Medio o Superior) & $31,4 \%$ \\
\hline & Estudios Universitarios & $12,8 \%$ \\
\hline
\end{tabular}

Tabla 2. Características sociodemográficas de la muestra

\subsection{Instrumento}

Para la recogida de la información se elabora un instrumento diseñado ad hoc, compuesto de una dimensión para cuestiones sociodemográficas (los 7 primeros ítems) y cuatro dimensiones que se relacionan con el contenido estudiado [Actuaciones de la Escuela (ACE), Información sobre Organización y Funcionamiento (IOF), Información Académica y Logros (IAL) y Relaciones Familia-Escuela (RFE)] y que aglutinan 13 ítems cerrados, de respuestas múltiples (4-5 opciones de respuesta) y organizados en dos tipos de escalas de respuestas: Likert ordinales (oscilando desde nunca hasta siempre) y nominales dicotómicas (Si/No) (McMillan y Schumacher, 2005). Para la construcción del instrumento se han considerado los fundamentos teóricos e instrumentos validados y evidenciados en la bibliografía consultada (Parra et al., 2017; Rodríguez, Villarreal y Danini, 2002; SINEACE, 2014; Valdés, Carlos y Arreola, 2013; Valdés, Martín y Sánchez, 2009).

Se procede a una validación de contenido a través de la técnica de juicio de expertos (claridad y adecuación de contenido) mediante la revisión de dos profesores de la Universidad de Huelva especialistas en metodología de investigación cuantitativa; asimismo, también se realiza una prueba piloto con 18 sujetos procedentes de la muestra seleccionada, a través de la cual se valora la dificultad de los ítems y el tiempo destinado a dar respuesta a estos. A partir de ambos casos, se realizaron ajustes menores 
relativos a la ortografía y la corrección de la formulación de 7 ítems, puesto que el tiempo aproximado (11 minutos) se consideró como adecuado. Para valorar la fiabilidad del instrumento se recurre a la prueba alfa de Cronbach y esta reporta un valor inicial de $\alpha=, 791$, pero atendiendo a los valores procedentes de la correlación ítem-total se advierte la posibilidad de eliminar el ítem "La Escuela programa reuniones periódicas con los docentes o tutores" puesto que correlaciona de forma negativa y se incrementa la fiabilidad hasta llegar a un valor de $\alpha=, 801$, con lo que de acuerdo con Oviedo-Heidi y Campo-Arias (2005), los valores de fiabilidad obtenidos a partir de la prueba Alfa de Cronbach son los que se prefieren en investigación.

\subsection{Procedimiento y análisis de la información}

La administración del instrumento tuvo lugar entre el 12 de mayo y el 8 de junio de 2019. Parte del desarrollo del trabajo de campo se implementó desde las prácticas de campo de la materia “Tutoría y Orientación en Educación Infantil” del primer curso del Grado de Educación Infantil de la Universidad de Huelva, disponiendo de la colaboración de los alumnos y las alumnas matriculados/as en la materia. Estos habían recibido formación en técnicas de recogida de datos como el cuestionario o las entrevistas con las familias $y$, organizados en equipos de trabajo, favorecieron el proceso de recogida de datos.

Como paso previo, a través de una notificación en papel se informó a los participantes acerca de los objetivos y sentido de la investigación que se estaba realizando, se solicitó su participación voluntaria y se garantizó el anonimato de las respuestas obtenidas. La distribución se produjo en los horarios y lugares acordados por los familiares (mayoritariamente hogar o espacios públicos).

Tras el proceso de recogida de información, se procedió a construir una base de datos con el programa Microsoft Excel bajo un fichero en formato CSV (Comma-SeparatedValues) en el que se tabularon las respuestas ofrecidas por la muestra participante. Esta base de datos fue usada con posterioridad para el tratamiento estadístico de la información con el software SPSSv17. Basándose en los objetivos marcados para el estudio se emplearon análisis descriptivos (frecuencias, tendencia central y dispersión) y correlaciones bivariadas (chi cuadrado, biserial, spearman).

\section{RESULTADOS}

A continuación, se exponen los resultados obtenidos en el análisis descriptivo de los ítems correspondientes a cada una de las dimensiones que estructuran el instrumento.

\subsection{Actuaciones de la Escuela (ACE)}

Los resultados obtenidos reflejan una respuesta afirmativa por parte de las familias en relación a la celebración por parte de los Centros educativos de aquellos eventos que son considerados habituales en las programaciones curriculares [fiestas y celebraciones $(95,5 \%)$; acciones que promueven la integración de estudiantes de distintas culturas, lenguas, niveles socio económicos, con discapacidad, etc. $(85,2 \%)$; reuniones académicas acerca del proceso educativo de sus hijos/as (79,5\%); reuniones 
informativas acerca de actividades u otra información relevante $(73,9 \%)$; y actividades extraescolares $(71,6 \%)$ ]. De la misma forma, también se exponen otros eventos en los que la opinión de la familia se muestra contraria a su desarrollo por parte de los centros educativos [convocatorias para participar en la toma de decisiones de los proyectos educativos $(61,4 \%)$; actividades deportivas $(60,9 \%)$; y talleres o prácticas para ampliar la formación de las familias $(52,3 \%)]$.

\subsection{Información sobre Organización y Funcionamiento (IOF)}

En cuanto al plano organizativo, las familias se muestran satisfechas y afirman que los centros han desarrollado iniciativas a partir de las cuales dar a conocer la institución, ya sea a nivel de organización y estructuración interna $(85,2 \%)$, informando de los roles que han desempeñar $(80,7 \%)$, mostrando las funciones particulares de los diferentes miembros (director, docentes, estudiantes, administrativos, etc.) (71,6\%) y dando a conocer los planes que tienen para mejorar los servicios que ofrecen $(69,3 \%)$.

La familia también reconoce que los Centros educativos se preocupan por mantenerles informados sobre el Proyecto Educativo en su conjunto, ya sea dando a conocer los proyectos que desarrollan (83,9\%), informando sobre la misión y la visión de la Institución (69\%), transmitiendo la propuesta pedagógica $(65,5 \%)$ y advirtiendo de los valores por los que se guían $(59,8 \%)$.

En líneas generales, el $72,4 \%$ de las familias señala que los centros educativos les mantienen informadas sobre los resultados, avances y dificultades a nivel de toda la institución.

\subsection{Información Académica y Logros (IAL)}

Atendiendo a la información ligada al plano académico-curricular, las respuestas que han ofrecido las familias a las opciones presentadas a modo de categorías, son muy positivas con respecto a la información facilitada por los Centros educativos y en todas y cada una de ellas (tomando en consideración los valores de la escala (mínimo= 1 , Máximo $=4$ ) la tasa de respuesta hacia una información muy continuada se sitúa por encima del $80 \%$ (Tabla 3 ).

Toda esta información relativa a factores asociados al proceso de enseñanza y de aprendizaje es facilitada por los Centros a través de diferentes canales, con los que las familias se encuentran altamente satisfechas. En concreto el $100 \%$ de la muestra encuestada se posiciona claramente a favor de los centros en los procesos de entrega de calificaciones de forma oportuna y en el desarrollo de entrevistas individuales cuando estas son solicitadas; y una muy amplia mayoría también reconocen que se programan reuniones periódicas con los docentes o tutores $(80,2 \%)$, y que toda la información que pueda surgir se les brinda por escrito (de manera física o virtual) $(81,4 \%)$. Así pues, la valoración hacia la frecuencia con la que reciben oportunidades de visitar a las maestras de sus hijos para debatir sobre su progreso (incluyendo las reuniones programadas) goza de una alta aprobación por parte de las familias $\left(\bar{x}=3,18 ; \mathrm{M}_{\mathrm{e}}=3,00 ; \mathrm{sd}=, 815\right)$. 


\begin{tabular}{|c|c|}
\hline Variables & Valores \\
\hline \multirow{3}{*}{ Lo que están aprendiendo mis hijos. } & $\bar{x}=3,6$ \\
\hline & $\mathrm{Me}=4,00$ \\
\hline & $\mathrm{sd}=, 637$ \\
\hline \multirow{3}{*}{ Los logros de mis hijos. } & $\bar{x}=3,62$ \\
\hline & $\mathrm{Me}=4,00$ \\
\hline & $\mathrm{sd}=, 703$ \\
\hline \multirow{3}{*}{ Las dificultades en el aprendizaje de mis hijos. } & $\bar{x}=3,67$ \\
\hline & $\mathrm{Me}=4,00$ \\
\hline & $\mathrm{sd}=, 584$ \\
\hline \multirow{3}{*}{ Lo que hacen los docentes para apoyar el aprendizaje de mis hijos. } & $\bar{x}=3,40$ \\
\hline & $\mathrm{Me}=4,00$ \\
\hline & $\mathrm{sd}=, 842$ \\
\hline \multirow{3}{*}{ La utilidad de lo que mis hijos están aprendiendo. } & $\bar{x}=3,30$ \\
\hline & $\mathrm{Me}=4,00$ \\
\hline & $\mathrm{sd}=, 941$ \\
\hline \multirow{3}{*}{ Me orienta sobre cómo apoyar el aprendizaje de mis hijos en casa. } & $\bar{x}=3,28$ \\
\hline & $\mathrm{Me}=3,00$ \\
\hline & $\mathrm{sd}=, 773$ \\
\hline \multirow{3}{*}{$\begin{array}{l}\text { Me brinda información sobre recursos o herramientas disponibles } \\
\text { para apoyar el aprendizaje de mis hijos. }\end{array}$} & $\bar{x}=3,18$ \\
\hline & $\mathrm{Me}=3,00$ \\
\hline & $\mathrm{sd}=, 829$ \\
\hline \multirow{3}{*}{$\begin{array}{l}\text { Tiene horarios disponibles para que los docentes y directivos } \\
\text { atiendan a los padres de familia. }\end{array}$} & $\bar{x}=3,51$ \\
\hline & $\mathrm{Me}=4,00$ \\
\hline & $\mathrm{sd}=, 745$ \\
\hline \multirow{3}{*}{$\begin{array}{l}\text { Me brinda información sobre los aspectos que se relacionan con el } \\
\text { aprendizaje de mis hijos (salud, alimentación, disciplina, etc.) }\end{array}$} & $\bar{x}=3,41$ \\
\hline & $\mathrm{Me}=4,00$ \\
\hline & $\mathrm{sd}=, 800$ \\
\hline
\end{tabular}

Tabla 3. Estadísticos descriptivos

\subsection{Relaciones Familia-Escuela (RFE)}

Para analizar los resultados pertenecientes a la dimensión relación familia-escuela, se atienden a tres factores esenciales. En primer lugar, se alude a la predisposición del centro para invitar a las familias para que participen en diferentes actividades aportadas por los investigadores; en este caso, se aprecia claramente cómo destacan las colaboraciones en las reuniones convocadas por el centro y por el profesorado y en menor medida cómo otras actividades ligadas al apoyo (educativo e institucional) son claramente poco extendidas hacia las familias (Tabla 4).

Esta disparidad en cuanto al tipo de invitación trasladada por los centros también se aprecia en una valoración general relativa a fomentar la participación en actividades para la mejora de la Institución (por ejemplo, cuidado del edificio, del medio ambiente, del trato entre personas, actividades hacia el aprendizaje, etc.), en las que solo el $62,1 \%$ de los encuestados reconocen haber recibido este tipo de invitación.

En segundo lugar, también se pone en valor el factor relativo a la consideración e importancia que los centros educativos dan a las opiniones de las familias. En este sentido, la opinión de la gran mayoría de las familias encuestadas (valores superiores al $85 \%$ ) entiende que los centros educativos poseen estrategias como las recogidas en la Tabla 5 con las que se garantiza una comunicación positiva y, por lo tanto, esta relación se fortalece. 


\begin{tabular}{ll}
\hline \multicolumn{1}{c}{ Variables } & \multicolumn{1}{c}{ Valores } \\
\hline \multirow{2}{*}{ Las reuniones de aula convocadas por el docente. } & $\bar{x}=3,35$ \\
& $\mathrm{Me}=4,00$ \\
& $\mathrm{sd}=, 898$ \\
\hline \multirow{2}{*}{ Las actividades artísticas, deportivas o celebraciones. } & $\bar{x}=3,17$ \\
\hline & $\mathrm{Me}=3,00$ \\
Las reuniones de padres de familia convocadas por la Escuela. & $\mathrm{sd}=, 874$ \\
\hline & $\bar{x}=3,50$ \\
Actividades de apoyo en el aula o la institución. & $\mathrm{Me}=4,00$ \\
& $\mathrm{sd}=, 788$ \\
\hline
\end{tabular}

Tabla 4. Estadísticos descriptivos

\begin{tabular}{|c|c|}
\hline Variables & Valores \\
\hline \multirow{3}{*}{ Considero que somos tratados con respeto. } & $\bar{x}=3,77$ \\
\hline & $\mathrm{Me}=4,00$ \\
\hline & $\mathrm{sd}=, 519$ \\
\hline \multirow{3}{*}{ Puedo expresar mis ideas y opiniones. } & $\bar{x}=3,57$ \\
\hline & $\mathrm{Me}=4,00$ \\
\hline & $\mathrm{sd}=, 621$ \\
\hline \multirow{3}{*}{$\begin{array}{l}\text { Se toma en cuenta mi opinión sobre la educación que se brinda a mis } \\
\text { hijos. }\end{array}$} & $\bar{x}=3,48$ \\
\hline & $\mathrm{Me}=4,00$ \\
\hline & $\mathrm{sd}=, 694$ \\
\hline \multirow{3}{*}{ Existen canales formales para comunicar y expresar mis opiniones. } & $\bar{x}=3,39$ \\
\hline & $\mathrm{Me}=4,00$ \\
\hline & $\mathrm{sd}=, 850$ \\
\hline \multirow{3}{*}{ Siento que valoran mi esfuerzo. } & $\bar{x}=3,41$ \\
\hline & $\mathrm{Me}=4,00$ \\
\hline & $\mathrm{sd}=, 768$ \\
\hline
\end{tabular}

Tabla 5. Estadísticos descriptivos

En tercer lugar, se indaga en el tipo de información que se demanda desde la escuela por parte del profesorado, tutores/as o desde las direcciones. En este caso, se aprecia que preocupa especialmente el entorno familiar (número de miembros, trabajo o actividad de los padres, costumbres familiares, etc., $81,8 \%$ ), seguida de las condiciones beneficiosas o no para el aprendizaje en el hogar (80,7\%); por otro lado, la familia reconoce una menor demanda de información por parte de los centros hacia aspectos más ligados a las preferencias de los estudiantes en cuanto al tipo de actividades que les gustan/interesan $(64,8 \%)$ y también hacia la organización del tiempo de los hijos en el hogar familiar $(55,7 \%)$.

Tras un primer análisis descriptivo, se procede a realizar las correlaciones necesarias para abordar los objetivos e hipótesis del trabajo. En este sentido es necesario precisar que no encontramos unas correlaciones altamente significativas entre las variables analizadas. Por el contrario, encontramos algunas correlaciones de poca intensidad:

En relación a la etapa educativa en la que las familias tienen matriculados a los estudiantes, con un nivel de confianza del $99 \%$ los resultados exponen una baja correlación $(r h o=, 286, p=, 008)$ entre esta variable y la celebración de reuniones informativas por parte de los centros sobre actividades u otra información relevante. Esta es una correlación que encuentra su sustento en algunas de las variables que 
componen los factores ligados a la relación Familia-Escuela (Tabla 6) y se deriva que, a medida que aumenta el nivel de estudios en el que están matriculados los estudiantes, la incidencia de las variables que componen los diferentes factores analizados disminuye y viceversa, por lo que situaríamos como principal foco de atención de los centros educativos aquellas familias que tienen los hijos matriculados en el primer ciclo de Educación Infantil.

\begin{tabular}{|c|c|c|c|}
\hline Factores & Variables & $\begin{array}{c}\text { (Rho de } \\
\text { Spearman) }\end{array}$ & $\begin{array}{c}\text { Sig. } \\
\text { (bilateral) }\end{array}$ \\
\hline \multirow{4}{*}{$\begin{array}{c}\text { La predisposición del } \\
\text { centro para invitar a las } \\
\text { Familias }\end{array}$} & Las reuniones de aula convocadas por el docente & rho $=-, 249$ & $\mathrm{p}=, 01$ \\
\hline & $\begin{array}{l}\text { Las actividades artísticas, deportivas o } \\
\text { celebraciones }\end{array}$ & rho $=-, 211$ & $\mathrm{p}=, 04$ \\
\hline & $\begin{array}{l}\text { Las reuniones de padres de familia convocadas } \\
\text { por la Escuela }\end{array}$ & rho $=-, 204$ & $\mathrm{p}=, 05$ \\
\hline & Actividades de apoyo en el aula o la institución & rho $=-, 240$ & $\mathrm{p}=, 02$ \\
\hline \multirow{3}{*}{$\begin{array}{c}\text { Consideración e } \\
\text { importancia hacia las } \\
\text { familias }\end{array}$} & Puedo expresar mis ideas y opiniones & rho $=-, 212$ & $\mathrm{p}=, 04$ \\
\hline & $\begin{array}{l}\text { Se toma en cuenta mi opinión sobre la educación } \\
\text { que se brinda a mis hijos }\end{array}$ & rho $=-, 317$ & $\mathrm{p}=, 003$ \\
\hline & Siento que valoran mi esfuerzo & rho $=-, 203$ & $\mathrm{p}=, 05$ \\
\hline \multirow{3}{*}{$\begin{array}{l}\text { Información que se } \\
\text { demanda desde la } \\
\text { escuela }\end{array}$} & Lo que están aprendiendo mis hijos & rho $=-, 316$ & $\mathrm{p}=, 003$ \\
\hline & Los logros de mis hijos & rho $=-, 366$ & $\mathrm{p}=, 000$ \\
\hline & Las dificultades en el aprendizaje de mis hijos & rho $=-, 213$ & $\mathrm{p}=, 04$ \\
\hline
\end{tabular}

Tabla 6. Correlaciones: coeficiente Rho de Spearman

$\mathrm{Si}$ atendemos a la variable relativa al tiempo de relación de las familias con los centros educativos, los resultados no muestran correlaciones significativas y solamente destaca que aquellas familias que presentan un menor tiempo de relación con los centros son a las que los profesores, tutores o la dirección, les requieren información sobre la situación del hogar o entorno que ayudan o dificultan su aprendizaje ( $r b p=, 259, p=$ ,015).

\section{DISCUSIÓN Y CONCLUSIONES}

Este trabajo tenía como finalidad contribuir al estudio de la interacción entre las familias y los centros de Educación Infantil mediante el empleo de diferentes medidas/acciones que flexibilizan el currículo (CCNSP, 2015; CEE, 2015; CEIP AM, 2015; Jurado-Gómez, 2009; MECD, 2014, 2015; Tamayo, 2014). Para alcanzar tal finalidad, se estableció como primer objetivo "analizar la opinión de las familias con hijos/as en la etapa de Educación Infantil para identificar en qué se focalizan las acciones flexibles que promueven los centros educativos"; a partir de los resultados expuestos puede evidenciarse una cotidianeidad en la utilización de diferentes acciones ligadas a aspectos en los que la familia ocupa un lugar de menor impacto en el currículo académico del alumno (fiestas y celebraciones, reuniones académicas e informativas, actividades extraescolares) (Castro-Zubizarreta y Manzanares-Ceballos, 2016; CCNSP, 2015; Ceballos-López y Saiz-Linares, 2019; MECD, 2014). Mientras que hay otros recursos en los que la familia puede tener más impacto en el currículo (toma de decisiones en los proyectos educativos) o en los que es destinataria de primer orden (talleres para la formación de las familias) y cuya utilización por parte de los centros 
educativos no se encuentra aún claramente consagrada (CCNSP, 2015; CEE, 2015; MECD, 2014).

Esta situación parece identificarse también en las acciones destinadas al conocimiento organizativo y funcional de las propias instituciones, existiendo así un mayor empleo de acciones destinadas hacia el conocimiento por parte de las familias de los elementos organizativos y estructurales y dejando ligeramente en un segundo plano aquellas iniciativas que se derivan del planteamiento pedagógico que se desarrolla en estos centros (MECD, 2014).

Al igual que sucede en otros trabajos (Arostegui, Darretxe y Beloki, 2013; CCNSP, 2015; Macia-Bordalba, 2017; Ruiz-De Miguel y García-García, 2004; Tamayo, 2014), y en la línea de lo descrito con anterioridad, las familias reconocen que las estrategias desarrolladas por los centros para trasladar toda la información relativa al plano más académico y próximo a los logros y los resultados de aprendizaje son adecuadas. Se evidencia con claridad la preocupación de los centros de Educación Infantil por mantener informadas a las familias sobre los temas ligados al desarrollo académico de sus hijos y para ello facilitan el contacto con los/as tutores/as y diversifican los canales de comunicación, ya sean presenciales o virtuales (entregas de calificaciones, entrevistas individuales, reuniones concertadas, etc.).

Por último, los datos apuntan a que a nivel de relación, las familias se consideran altamente satisfechas con el trato recibido por los centros educativos (respeto, libertad de expresión, diversidad de canales de comunicación, etc.) pero sus respuestas denotan que falta una mayor disponibilidad o predisposición por parte de los centros educativos para generar una relación más intensa a través de diferentes acciones que les faciliten una mayor presencia y decisión en aspectos más próximos al plano pedagógico (actividades de apoyo en el aula o la institución) y al institucional (actividades artísticas, deportivas o celebraciones) (Ortega-Ruiz, Romera y Monks, 2013; Payá-Rico, 2010; Vendrell et al., 2019), así como otras decisiones que demuestren también un interés hacia los intereses personales de las propias familias, de los estudiantes y de cómo se distribuyen los tiempos en el hogar (Dail y McGee, 2008; MECD, 2014; SierraMartínez, 2018).

Como segundo objetivo, se propuso "determinar la influencia de las variables “tiempo de relación con el centro" y "nivel educativo de los estudiantes" en relación a las consideraciones sobre la flexibilidad curricular de los centros educativos". Atendiendo a los resultados descritos no se encuentran correlaciones altamente significativas entre las variables indicadas, aunque sí que se intuye que el nivel de estudios encuentra una correlación negativa con respecto al grado de implicación o la presencia de las familias en las diferentes iniciativas que los centros diseñan e implementan tal y como señala la literatura (Garreta, 2008; Gomariz et al., 2008; MECD, 2014, 2015; Paniagua-Valle, 2009; Parra et al., 2017; Peñalver-Pérez, 2009); algo similar ocurre con la variable relativa al tiempo que una familia mantiene contacto con el centro por la matriculación de sus hijos en el mismo, se intuye que los centros educativos implementan más acciones hacia el conocimiento de las familias que llevan menos tiempo de cara a obtener una información que sea beneficiosa para el proceso de enseñanza y aprendizaje del estudiante.

A partir de estas consideraciones y valorando con cautela los resultados obtenidos podríamos afirmar la $\left(\mathrm{H}_{1}\right)$ aunque se aprecia que es necesario seguir promoviendo iniciativas que ayuden a flexibilizar el currículo de la etapa de infantil para lograr que 
las familias tengan un participación mucho más ágil y factible con los centros educativos. En la misma línea, también se acepta la $\left(\mathrm{H}_{2}\right)$ y se aprecia cómo las familias reconocen una mayor utilización por parte de los centros educativos de acciones diversificadas y próximas a otorgar información sobre el centro y sobre el proceso de enseñanza y aprendizaje. Por otra parte, para la aceptación de las $\left(\mathrm{H}_{3}\right)$ y $\left(\mathrm{H}_{4}\right)$ no hemos logrado encontrar evidencias que así lo corroboren, y solo se han determinado posibles correlaciones débiles entre las variables estudiadas, quizás destaca con más significatividad la variable nivel de estudios (hijos matriculados más pequeños o más mayores) como elemento que se relaciona con la mayor o menor cantidad de acciones curriculares flexibles empleadas por los centros para interaccionar con las familias.

Como conclusiones, hay que destacar que es primordial que tanto la familia como el centro educativo deban afrontar la educación de los niños de forma coordinada, complementaria, de colaboración y teniendo en cuenta que es desde la etapa de Educación Infantil donde las familias tienen más presencia en el centro educativo. Por ello, es necesario establecer desde un principio una serie de medidas y/o acciones que flexibilicen el currículo, pero no solo en aquellas actividades que impacten en menor grado en el currículo, sino que hay que priorizar más la presencia de la familia en actividades académicas oficiales. Pero esto no quiere decir que con la participación de las familias en el Consejo Escolar, el AMPA, etc. se resuelva esta problemática, sino que las familias en todo momento deben de intervenir, sin obviar el intrusismo, en las iniciativas que se deriven del planteamiento pedagógico de los centros educativos.

Estudios como los citados en este trabajo afirman que las familias están de acuerdo con las estrategias educativas que se llevan a cabo en el centro educativo en cuanto a la información de los resultados y logros de cada uno de los niños, y que se pueden llevar a cabo de forma unidireccional o de forma bidireccional en función de la preocupación del propio centro. También están enormemente satisfechas con las conexiones entre la familia y el centro, una relación que decae cuando son actividades más de carácter pedagógico.

Existen pocos estudios (Vendrell, Dalmau, Gallego y Baqués, 2015) que confirmen que las familias del primer ciclo de Educación Infantil participen más que el segundo ciclo. En esa investigación, sobre los varones como profesionales de la Educación Infantil, se realizó una diferencia entre la participación de las familias entre 0-3 años (17\% de participación) y 3-6 años (9\%). El resto de estudios claramente evidencian un descenso en la participación de las familias a medida que se escala en las diferentes etapas educativas.

En definitiva, es imprescindible seguir ahondando en las relaciones entre la familia y el centro educativo a través de iniciativas que flexibilicen el currículo, que sean informadas mediante acciones que tengan en consideración el proceso de enseñanza y aprendizaje del alumnado y con actividades de índole formal y no formal para que el rendimiento del alumnado sea óptimo.

Como limitaciones del presente estudio debe considerarse que los hallazgos expuestos en este trabajo no pueden ser inferenciados al universo de referencia por lo que se sugiere seguir ahondando en la opinión de las familias hacia las acciones curriculares flexibles que emplean los centros que dan una respuesta educativa en la etapa de infantil, por ejemplo, ampliando los tamaños muestrales de tal manera que la información recogida sea más representativa. También sería de interés seguir trabajando en la construcción de un instrumento sobre satisfacción de las familias con el objeto de 
estudio de manera que arrojara indicadores de validez, coeficientes de fiabilidad, varianza explicada y capacidad predictiva más adecuados. De este aspecto surge la posibilidad de realizar modelos de ecuaciones estructurales para evaluar la validez de medida y analizar los índices de bondad de ajuste. Finalmente, para completar la información recogida en este estudio sería conveniente ampliar el trabajo hacia un diseño de investigación mixto, que combinara estrategias cuantitativas y cualitativas con las que obtener una visión más exhaustiva y explicativa sobre la satisfacción que tienen las familias hacia la respuesta curricular flexible de los centros que imparten Educación Infantil.

\section{BIBLIOGRAFÍA}

Alves Martins, M., Salvador, L., Albuquerque, A. y Montanero Fernández, M. (2017). “Otro niño lo escribió así”. Ayuda educativa y resultados de actividades de escritura inventada. Revista de Educación, 377, 161-186. http://dx.doi.org//10.4438/1988-592X-RE-2017-377-357

Alzubi, T., Fernández, R., Flores, J., Durán, M. y Cotos, M. (2018). Incremento de las capacidades ejecutivas mediante el uso de herramientas interactivas basadas en gestos: caso de estudio la memoria de trabajo. Proceedings of XIX International Conference on HumanComputer Interaction (AIPO). Libro de Actas (pp. 1-6). Mallorca, España. http://dx.doi.org//10.1145/3233824.3233826

Amar, V. (2018). Déjame que mire un cuento: Narración, familia y educación infantil. Una investigación narrativa. Revista de Currículum y Formación del Profesorado, 22(2), 389405. https://doi.org/10.30827/profesorado.v22i2.7729

Arostegui, I., Darretxe, L. y Beloki, N. (2013). La participación de las familias y de otros miembros de la comunidad como estrategia de éxito en las escuelas. Revista Iberoamericana de Evaluación Educativa, 6(2), 187-200. Recuperado de: https://cutt.ly/Xfd4vc3

Aristizábal, P., Lasarte, G. y Tresserras, A. (2015). Jugar con las imágenes: alfabetización audiovisual en la Educación Infantil. Revista de Investigación en Educación, 13(2), 243255. Recuperado de: https://cutt.ly/Bfd4YKK

Arranz Freijo, E.B., Olabarrieta Artetxe, F., Manzano Fernández, A., Martín Ayala, J.L. y Galende Pérez, N. (2012). Escala Etxadi Gangoiti: una propuesta para evaluar los contextos familiares de niños de 2 años de edad. Revista de Educación, 358, 218-237. http://dx.doi.org//10-4438/1988-592X-RE-2010-358-076

Bronfenbrenner, U. (1979). The Ecology of Human Development. Cambridge: Harvard University Press.

Bronfenbrenner, U. (2005). Making Humans Being Human. Bioecological Perspectives on Human Development. London: Sage Publications.

Burns, R.B. (1990). El autoconcepto: Teoría, Medición, Desarrollo y Comportamiento. Bilbao: EGA.

Castro Zubizarreta, A. y Manzanares Ceballos, N. M. (2016). Los más pequeños toman la palabra: la Escuela Infantil que a ellos les gustaría. Revista Complutense de Educación, 27(3), 923-941. http://dx.doi.org/10.5209/rev_RCED.2016.v27.n3.47004

Ceballos López, N. y Saiz Linares, A. (2019). La acción tutorial como escenario de colaboración familia-escuela. REOP, 30(2), 28-45.

https://doi.org/10.5944/reop.vol.30.num.2.2019.25336

CEIP Antonio Machado (2015). Transformando el centro, transformando el barrio. Participación Educativa, Revista del Consejo Escolar del Estado, 4(7), 101-110. Recuperado de: https://cutt.ly/Yfd4ZDk 
Cid Lucas, F. (2009). El "Kamishibai” como recurso didáctico en el aula de Educación Infantil y Primaria. Bordón, 61(4), 141-149. Recuperado de: https://cutt.ly/0fd4N10

Colegio Concertado Nuestra Señora de la Providencia (2015). Escuela de familias: nos educamos juntos. Participación Educativa, Revista del Consejo Escolar del Estado, 4(7), 93-100. Recuperado de: https://cutt.ly/Qfd40Kh

Consejo Escolar del Estado (2015). Las relaciones entre familia y escuela. Experiencias y buenas prácticas. Encuentro de Consejos Escolares Autonómicos y del Estado. Madrid: Ministerio de Educación, Cultura y Deporte.

Dail, A.R. y McGee, L.M. (2008). Transition to kin-dergarten: Reaching back to preschoolers and parents through shared summer school. Childhood Education, 84(5), 305-311. https://doi.org/10.1080/00094056.2008.10523031

Da Silva Filgueira, M.G. y González González, C.S. (2017). PequeBot: Propuesta de un Sistema Ludificado de Robótica Educativa para la Educación Infantil. V Congreso Internacional de Videojuegos y Educación (CIVE'17). Tenerife, España: Universidad de la Laguna.

De Vicente-Yagüe Jara, M.I. y González Romero, M. (2019). Análisis de un planteamiento metodológico interdisciplinar en Educación Infantil para el fomento de la lectura. Revista Complutense de Educación, 30(2), 493-508. http://dx.doi.org/10.5209/RCED.57738

Doval, M.I., Martínez-Figueira, M.E. y Raposo, M. (2013). La voz de sus ojos: la participación de los escolares mediante Fotovoz. Revista de Investigación en Educación, 11(3), 150-171. Recuperado de: https://cutt.ly/efd7q4S

Durán, M., Álvarez, T., Fernández, R., y Acuña, A. (2015). Eficacia de un entrenamiento en funciones ejecutivas sobre las habilidades matemáticas básicas y la conciencia fonológica en niños de educación infantil. Revista de Estudios e Investigación en Psicología y Educación, Extra(9), 104-108. http://dx.doi.org/10.17979/reipe.2015.0.09.1158

Egido Gálvez, I. (2015). Las relaciones entre familia y escuela. Una visión general. Participación Educativa, Revista del Consejo Escolar del Estado, 4(7), 11-18. Recuperado de: https://cutt.ly/zfd7ynh

El Ouarit, M., Bouhachi, O. y Eddaif, Z. (2007). Educación infantil: 5 años. La casa y la familia. (El perro de Mariam). Lengua árabe. Materiales de trabajo del alumno. España: Secretaría General Técnica. Centro de Publicaciones. Ministerio de Educación y Ciencia.

Esteban-Guitart, M., Oller, J. y Vila, I. (2012). Vinculando escuela, familia y comunidad a través de los fondos de conocimiento e identidad. Un estudio de caso con una familia de origen marroquí. Revista de Investigación en Educación, 10(2), 21-34. Recuperado de: https://cutt.ly/Cfd7oHQ

Fernández-Abella, R., Peralbo-Uzquiano, M., Durán-Bouza, M., Brenlla-Blanco, J.C. y GarcíaFernández, M. (2019). Programa de intervención virtual para mejorar la memoria de trabajo y las habilidades matemáticas básicas en Educación Infantil. Revista de Psicodidáctica, 24(1), 17-23. https://doi.org/10.1016/j.psicod.2018.09.002

Franco Justo, C. y Justo Martínez, E. (2010). «Barrio Sésamo» y creatividad infantil: efectos de un programa psicoeducativo. Bordón, 62(4), 81-94. Recuperado de: https://cutt.ly/jfd7sdN

García Hernández, F. (2003). La orientación psicopedagógica en la Educación Infantil y su repercusión en el desarrollo del conocimiento de los niños de esta etapa. Revista Complutense de Educación, 14(1), 231-262. Recuperado de: https://cutt.ly/Pfd7go0

Garreta, J. (2008). La participación de las familias en la escuela pública. Las Asociaciones de Madres y Padres del Alumnado. Madrid: CEAPA.

Gomariz, M.A., Parra, J., García-Sanz, M. P., Hernández, M.A. y Pérez, J. (2008). La comunicación entre la familia y el centro educativo. Murcia: Consejo Escolar de la Región de Murcia, Consejería de Educación, Ciencia e Investigación.

González González, C.S. (2019). Estado del arte en la enseñanza del pensamiento computacional y la programación en la etapa infantil. Education in the Knowledge Society, 20, 15-28. https://doi.org/10.14201/eks2019_20_a17 
González-Valenzuela, M.J., Martín Ruiz, I., Prieto Adánez, G. y Rivas-Moya, T. (2018). Análisis del rendimiento y de la mejora en la Lectura y en la Escritura en Educación Infantil. Revista de Educación, 382, 225-247. https://doi.org/10.4438/1988-592X-RE-2018-382-398

Hernández Prados, M.A., Gomariz Vicente, M.A., Parra Martínez, J. y García Sanz, M.P. (2015). El sentimiento de pertenencia en la relación entre familia y escuela. Participación Educativa, Revista del Consejo Escolar del Estado, 4(7), 49-56. Recuperado de: https://cutt.ly/sfd7WUN

Hernández Prados, M.A., Gomariz Vicente, M.A., Parra Martínez, J. y García Sanz, M.P. (2016). Familia, inmigración y comunicación con el centro escolar: Un estudio comparativo. Educación XXI, 19(2), 127-151. https://doi.org/10.5944/educXX1.16457

Ibarra-Rius, N. y Ballester-Roca, J. (2016). Booktrailer en Educación Infantil y Primaria: adquisición y desarrollo de las competencias comunicativa, digital y literaria a través de narrativas digitales. Digital Education Review, 30, 76-93. Recuperado de: https://cutt.ly/dfd7YW1

Jover, G. y Payá Rico, A. (2013). Juego, educación y aprendizaje. La actividad lúdica en la pedagogía infantil. Bordón, 65(1), 13-18. https://doi.org/10.13042/brp.2013.65100

Jurado Gómez, C. (2009). La familia y su participación en la comunidad educativa. Innovación y Experiencias Educativas, 23, 1-10. Recuperado de: https://cutt.ly/Tfd7IMi

Laboratorio Permanente de Público de Museos (2016). Conociendo a todos los públicos. Un análisis de la visita al museo en familia. España: Secretaría General Técnica. Subdirección General de Documentación y Publicaciones. Ministerio de Educación, Cultura y Deporte.

Macia Bordalba, M. (2017). Principales canales para la comunicación familia-escuela: análisis de necesidades y propuestas de mejora. Revista Complutense de Educación, 30(1), 147-165. http://dx.doi.org/10.5209/RCED.56034

McMillan, J.H. y Schumacher, S. (2005). Investigación educativa. Una introducción conceptual. Madrid: Pearson Educación, S. A.

Medina Fuentes, M.J. (2019). Somos dioses egipcios. Conociéndonos a través de las TIC en Educación Infantil. Experiencias Educativas inspiradoras, 24, 1-16. http://dx.doi.org/10.4438/2695-4184_EEIpdf24_2020_847-19-133-8

Ministerio de Educación, Cultura y Deporte (2014). La participación de las familias en la educación escolar. Mirando al futuro. España: Secretaría General Técnica. Subdirección General de Documentación y Publicaciones. Ministerio de Educación, Cultura y Deporte.

Ministerio de Educación, Cultura y Deporte (2015). Las relaciones entre familia y escuela. Experiencias y buenas prácticas. España: Secretaría General Técnica. Subdirección General de Documentación y Publicaciones. Ministerio de Educación, Cultura y Deporte.

Molinero Maldivo, R. (2019). Desarrollando la CreApptividad en Educación Infantil. Experiencias Educativas Inspiradoras, 20, 1-16. http://dx.doi.org/104438/26954184_EEIpdf20_2019_847-19-133-8

Ortega-Ruiz, R., Romera, E.M. y Monks, C.P. (2013). La potencialidad interactiva en aulas de educación infantil en función del estatus sociométrico y del tipo de actividad. Bordón, 65(1), 119-130. https://doi.org/10.13042/brp.2013.65108

Oviedo Heidi, C. y Campo-Arias, A. (2005). Aproximación al uso del coeficiente alfa de Cronbach. Revista Colombiana de Psiquiatría, 34(4), 572-580. Recuperado de: https://bit.ly/330x2Ip

Paniagua Valle, G. (2009). El desarrollo de la Educación Infantil: un crecimiento costoso. CEE Participación Educativa, 12, 20-34. Recuperado de: https://cutt.ly/8fd7Fo6

Pardo Artero, M. (2019). Mapamundi interactivo de animales. Chroma Key en Educación Infantil. Experiencias Educativas Inspiradoras, 17, 1-15. https://doi.org/104438/2695-4184_EEIpdf17_2019_847-19-133-8

Parra, J., Gomariz, M.A., Hernández-Prados, M.A. y García-Sanz, M.P. (2017). La participación de las familias en educación infantil. RELIEVE, 23(1), art. 4.

http://doi.org/10.7203/relieve.23.1.9258 
Payá Rico, A. (2010). Consideraciones pedagógicas sobre los valores y posibilidades educativas del juego en la España contemporánea (1876-1936). Historia de la Educación, 26, 299-325. Recuperado de: https://cutt.ly/hfd7Lnc

Peñalver Pérez, R. (2009). El Plan Educa3, apostando por el primer ciclo de Educación Infantil. CEE Participación Educativa, 12, 8-19. Recuperado de: https://cutt.ly/1fd7XOP

Rideout, V, Vandewater, E. y Wartella, E. (2003). Zero to six. Electronic media in the lives of infants, toddlers and preschoolers. A Kaiser Family Foundation report. Washington, DC: The Henry Kaiser Family Foundation

Rivas, S. y Ugarte, C. (2014). Formación docente y cultura participativa del centro educativo: claves para favorecer la participación familia-escuela. Estudios sobre Educación, 27, 153168. http://doi.org/10.15581/004.27.153-168

Robles Meléndez, W. (2012). Tecnología en el aula infantil. Apuntes y comentarios. Revista Complutense de Educación, 23(1), 149-160. https://doi.org/10.5209/rev_RCED.2012.v23.n1.39107

Rodríguez, R, Villarreal, A. y Danini, J. (2002). Encuesta para padres y madres de familia de instituciones educativas públicas. San Antonio (Texas): Intercultural Development Research Association.

Ruiz de Miguel, C. (1999). La familia y su implicación en el desarrollo infantil. Revista Complutense de Educación, 10(1), 289-304. Recuperado de: https://cutt.ly/Ufd7BOk

Ruiz de Miguel, C. y García García, M. (2004). Modelo explicativo de factores vinculados a la calidad en educación infantil. Revista de Investigación Educativa, 22(2), 497-518. Recuperado de: https://cutt.ly/qfd7NJK

Sampedro Requena, B.E., Muñoz González, J.M. y Vega Gea, E. (2017). El videojuego digital como mediador del aprendizaje en la etapa de Educación Infantil. Revista Educar, 53(1), 89-107. https://doi.org/10.5565/rev/educar.850

Santabárbara Bayo, D. (2019). Valdespartera es cultura. 3D y realidad aumentada en Educación Infantil. Experiencias Educativas inspiradoras, 7, 1-14. https://doi.org/104438/26954184_EEIpdf7_2019_847-19-133-8

Santos Rego, M.A. y Slavin, R.E. (2002). La condición del éxito en la intervención pedagógica con niños en situación de riesgo: el programa "Success for All". Revista de Investigación Educativa, 20(1), 173-188. Recuperado de: https://cutt.ly/hfd71 Nz

Serrano Muñoz, A., Mérida Serrano, R. y Tabernero Urbieta, C. (2016). La autoestima infantil, la edad, el sexo y el nivel socioeconómico como predictores del rendimiento académico. Revista de Investigación en Educación, 14(1), 53-66. Recuperado de: https://cutt.ly/Ufd725t

Sierra Martínez, S. (2018). Investigaciones sobre la Transición a Educación Primaria: la mirada infantil a examen. Revista de Investigación en Educación, 16(2), 136-152. Recuperado de: https://cutt.ly/7fd73xU

Sistema Nacional de Evaluación, Acreditación y Certificación de la Calidad Educativa (SINEACE) (2014). Encuesta para padres y madres de familia de instituciones educativas públicas. Perú: Ministerio de Educación. Recuperado de: https://cutt.ly/4fd78Pr

Tamayo, S. (2014). La transición entre etapas educativas: de Educación Infantil a Educación Primaria. Participación Educativa, Revista del Consejo Escolar del Estado, 3(5), 130-137. Recuperado de: https://cutt.ly/efd74Sy

Valdés, Á.A., Martín, M. y Sánchez, P.A. (2009). Participación de los padres de alumnos de educación primaria en las actividades académicas de sus hijos. Revista Electrónica de Investigación Educativa, 11(1). Recuperado de: https://cutt.ly/1fd77De

Valdés, A., Carlos, E. y Arreola, C. (2013). Desarrollo de un instrumento para medir la participación de los padres en la educación escolar de los hijos. Revista de Evaluación Educativa, 2(1), 92-109. Recuperado de: https://cutt.ly/kfd75Hl 
Vendrell- Mañós, R., Dalmau, M., Gallego, S. y Baqués, M. (2015). Los varones, profesionales en la educación infantil. Implicaciones en el equipo pedagógico y en las familias. Revista de Investigación Educativa, 33(1), 195-210. http://dx.doi.org/10.6018/rie.33.1.184051

Vendrell-Mañós, R., Geis Balagué, Á., Anglès Virgili, N. y Dalmau Montalà, M. (2019). Percepción de los maestros sobre el derecho al juego libre en educación infantil y educación primaria. Estudio desarrollado en Barcelona (España). Bordón, 71(4), 151-165. https://doi.org/10.13042/Bordon.2019.71548

Zabalza, M.A. y Formosinho, J. (1998). Aspectos claves de una educación infantil de calidad. En M.D. García Fernández (Coord.), La educación infantil: investigación y desarrollo del currículum (pp. 119-124). Córdoba: Universidad de Córdoba. Servicio de Publicaciones. 